\title{
Laser Guide Star Objective of MAORY
}

\author{
M. Lombini ${ }^{\text {a, }}$, M. Patti ${ }^{\mathrm{a}, \mathrm{b}}$, C. Arcidiacono ${ }^{\mathrm{a}}$, P. Ciliegi ${ }^{\mathrm{a}}$, F. Cortecchia ${ }^{\mathrm{a}}$, A. De Rosa ${ }^{\mathrm{c}}$, E. Diolaiti $^{\mathrm{a}}$, \\ P. Feautrier ${ }^{\mathrm{d}}$, M. Moschetti ${ }^{\mathrm{e}}$, S. Oberti ${ }^{\mathrm{f}}$, P. Rabou ${ }^{\mathrm{d}}$, M. Riva ${ }^{\mathrm{e}}$, E. Stadler ${ }^{\mathrm{d}}$. \\ ${ }^{a}$ INAF - Osservatorio Astronomico di Bologna, Via Piero Gobetti 93/3, 40129 Bologna, Ialy \\ ${ }^{\text {b}}$ Alma Mater Studiorum, Università di Bologna, Dipartimento di Fisica e Astronomia, Via Gobetti 93/2, 40129 Bologna, \\ Italy; \\ ${ }^{c}$ INAF-Istituto di Astrofisica Spaziale e Fisica Cosmica, Via Gobetti 101, 40129 Bologna (Italy); \\ ${ }^{\mathrm{d}}$ Institut de Planétologie et d'Astrophysique de Grenoble, 414, Rue de la Piscine, Domaine Universitaire, 38400 St- \\ Martin d'Hères (France) \\ ${ }^{\text {e} I N A F-~ O s s e r v a t o r i o ~ A s t r o n o m i c o ~ d i ~ B r e r a, ~ V i a ~ E . ~ B i a n c h i ~ 46, ~} 23807$ Merate (Italy); \\ ESO, Karl-Schwarzschild-Str. 2, 85748 Garching bei München, Germany
}

\begin{abstract}
The Multi Conjugate Adaptive Optics Relay (MAORY) for the European Extremely Large Telescope is planned to be located on the straight-through port of the telescope Nasmyth platform and shall re-image the telescope focal plane to a wide field camera (MICADO) and a possible future second instrument. By means of natural and artificial (laser) reference sources for wavefront sensing, and of deformable mirrors for wavefront correction, MAORY shall be able to compensate the wavefront disturbances affecting the scientific observations, achieving high Strehl ratio and very low geometric distortion.

Inside the Post Focal Relay, the light at 589nm of the 6 sodium laser sources will be split from the longer wavelength light by a dichroic placed close to a pupil image and focused, by means an objective, into an image plane where the wavefront sensors will be placed. The image plane of the laser stars will shift along the optical path due to the variation of the Sodium layer distance with the Zenith angle. The objective shall maintain fixed the exit pupil position and the focal aperture for the different altitudes. Moreover the non-common path wavefront error and the pupil image optical quality should not affect significantly the wavefront reconstruction. We present in this paper the optical design and the tolerance analysis of the objective.
\end{abstract}

Keywords: Extremely Large Telescope, Multi-conjugate Adaptive Optics, Tolerance analysis

\section{INTRODUCTION}

MAORY [1][2] will be the multi-conjugate adaptive optics module for the Extremely Large Telescope (ELT) [3] first light. MAORY is a post focal relay optics and has to enable high performance astronomical observations in particular by the MICADO [4] (MCAO Imaging Camera for Deep Observations) imager and spectrograph. At first light, MAORY will contain a single deformable mirror with provision for a second deformable mirror as an upgrade. In the MCAO mode, at least one deformable mirror within MAORY works together with the telescope M4. The two or more deformable mirrors (DMs) are conjugated to different altitudes to provide a wide field of view with high Strehl ratio and uniformity of the point spread function (PSF). The SCAO mode will deliver high Strehl ratio for a smaller field, rather than uniform Strehl ratio over a wide field. To deliver the performance required by the MICADO science case, MAORY uses six laser guide star (LGS) wavefront sensors (WFS) and three natural guide star (NGS) wavefront sensors in the MCAO mode, while the SCAO mode, as defined, works on a single bright star.

The current baseline optical design of MAORY is based on six mirrors (including one or two adaptive mirrors) and a dichroic beam-splitter. The layout of the Post-Focal Relay Optics is shown in

*matteo.lombini@oabo.inaf.it 


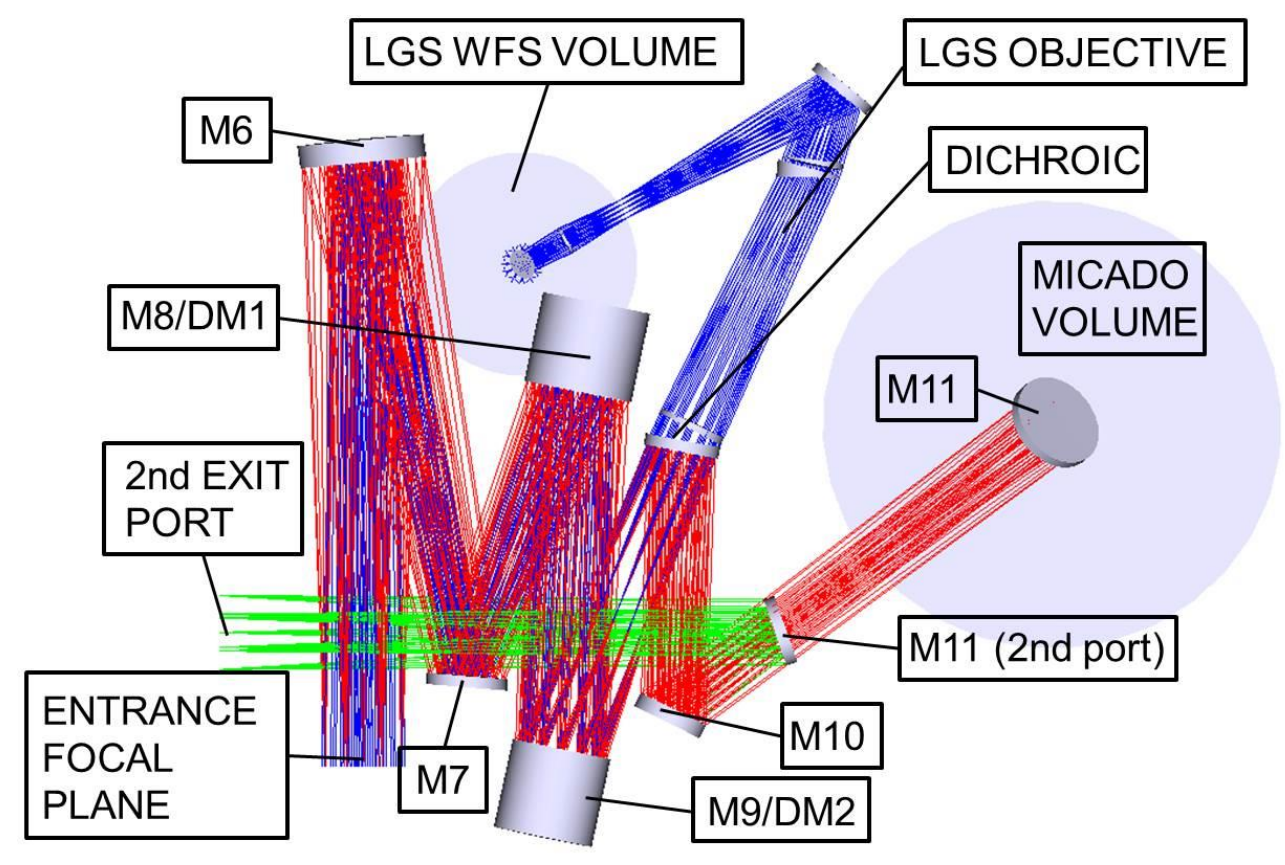

Figure 1. Post-Focal Relay Optics layout. Red rays: optical beam from telescope focal plane to exit port for MICADO. Green rays: differential path to exit port for second instrument. Blue rays: LGS path (shown in Figure 2). The light-grey circles in the background are the projection of the design volumes for the LGS WFS and for MICADO.

\section{LGS OBJECTIVE DESIGN}

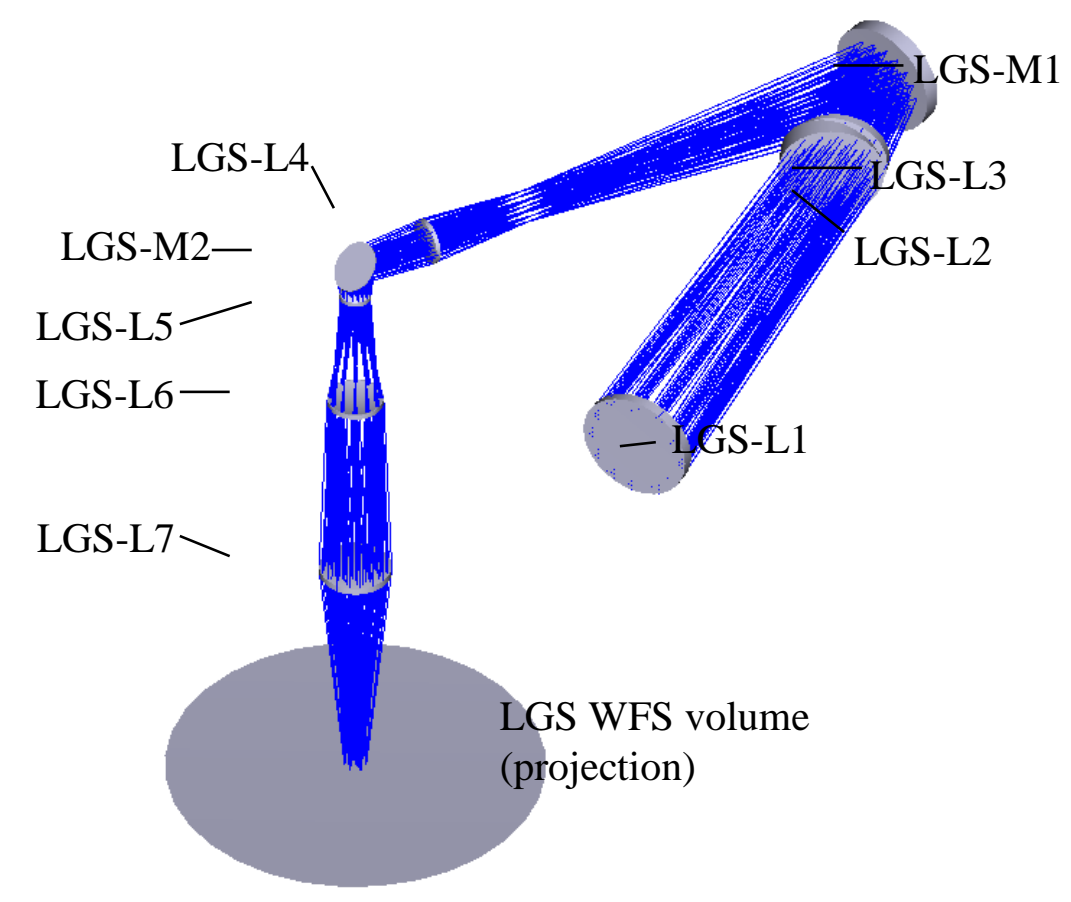

Figure 2. LGS Objective layout. 
The LGS light propagated through mirrors M6, M7, M8 (DM) and M9 (DM) is transmitted by the LGS Dichroic, which is located closed to a pupil plane, and is focused by the LGS Objective to create an image of the 6 LGSs for the LGS WFS sub-system.

The general specifications of the LGS Objective are reported in the next table.

\begin{tabular}{|l|l|}
\hline Item & Value \\
\hline Operating wavelength & $0.589 \mu \mathrm{m}$ \\
\hline LGS range to be re-imaged & $84-240 \mathrm{~km}$ \\
\hline Exit focal ratio & $\mathrm{F} / 5$ \\
\hline Exit pupil distance & Infinity \\
\hline LGS constellation maximum angular diameter & 120 arcsec \\
\hline
\end{tabular}

Table 1. LGS Objective Optics general specifications.

The LGS Objective is designed to deliver LGS images in the altitude range $84-240 \mathrm{~km}$. The output focal ratio of F/5 allows reasonable motion of the exit image plane, as a function of Zenith distance variation during the observation.

The 6 LGSs are supposed to be launched form the edge of the primary mirror and thus they rotate as the Zenith angle of observation. Their image plane shifts along the optical axis due to the variation of the mean distance of the LGS from the telescope, as $\mathrm{h}_{\mathrm{Na}} / \cos (\theta)$, where $\mathrm{h}_{\mathrm{Na}} \approx 90 \mathrm{~km}$ is the Sodium layer mean altitude above the ground.

The LGS Objective, shown in Figure 2, consists of three wedged lensed (the wedge is on a plane surface - so that these lenses may in principle be replaced by normal lenses plus wedges), a powered mirror, a flat mirror and four lenses. The baseline material for all refractive elements is optical glass (BK7, S-BSL7 or equivalent glass).

The LGS Objective contains an intermediate image plane and an intermediate pupil plane. The last part of the optical axis is folded by the flat mirror LGS-M2, so that the LGS WFS sub-system is mounted in gravity invariant configuration. The next table shows the prescription data of the optical elements in the LGS Objective. Detailed performance and tolerance analysis of the LGS Objective is shown in section 3.

\begin{tabular}{|c|c|c|c|c|c|}
\hline ID & $\begin{array}{r}\text { Diameter } \\
{[\mathrm{mm}]}\end{array}$ & $\begin{array}{r}\text { Thickness } \\
\text { [mm] }\end{array}$ & $\begin{array}{r}\text { Radius } \\
\text { [mm] }\end{array}$ & $\begin{array}{r}\text { Conic } \\
\text { constant } \\
{[1]}\end{array}$ & Wedge \\
\hline LGS-L1 & 660 & 61 & $\begin{array}{r}\text { Infinity } \\
\text { (cx) } 8946\end{array}$ & $\begin{array}{r}- \\
-10.000\end{array}$ & yes \\
\hline LGS-L2 & 600 & 55 & $\begin{array}{r}\text { Infinity } \\
\text { (cv) } 3234\end{array}$ & - & yes \\
\hline LGS-L3 & 580 & 55 & $\begin{array}{r}\text { (cx) } 1830 \\
\text { Infinity }\end{array}$ & -0.827 & yes \\
\hline LGS-M1 & 620 & - & (cv) 23516 & -4.605 & - \\
\hline LGS-L4 & 380 & 41 & $\begin{array}{r}\text { (cx) } 1585 \\
\text { (cx) } 641\end{array}$ & $\begin{array}{r}- \\
-4.709\end{array}$ & - \\
\hline LGS-M2 & 420 & - & flat & - & - \\
\hline LGS-L5 & 240 & 21 & $\begin{array}{r}(\mathrm{cx}) 109 \\
(\mathrm{cv}) 76\end{array}$ & $\begin{array}{l}-1.258 \\
-1.007 \\
\end{array}$ & - \\
\hline LGS-L6 & 340 & 48 & $\begin{array}{l}\text { (cv) } 666 \\
\text { (cx) } 331\end{array}$ & $\begin{array}{l}-0.018 \\
-0.260 \\
\end{array}$ & - \\
\hline LGS-L7 & 420 & 63 & $\begin{array}{l}\text { (cx) } 1376 \\
\text { (cx) } 1475\end{array}$ & $\begin{array}{l}-1.625 \\
-2.866\end{array}$ & - \\
\hline
\end{tabular}

Table 2. Prescription data of optical elements in the LGS Objective. When two values are contained inside a single row, they refer to the front and rear surface of the optical element. 


\subsection{LGS Objective wavefront error}

The performance of the LGS Objective is shown in the next figures. Figure 3 shows the WFE map over the possible range of LGS constellation angular diameter, for three different cases of Zenith angle. Figure 4 shows the WFE vs. Zenith angle (after removal of tip-tilt and focus). The variation of wavefront with Zenith angle is very slow. The calibration procedure to remove these quasi-static aberrations from the LGS measurements might be based on temporal filtering of the LGS WFS measurements. Figure 5 shows the variation of residual WFE vs. Zenith angle (after removal of tip-tilt and focus), assuming that the average wavefront computed over the last 4 minutes in time is subtracted from the wavefront at a given Zenith angle. The residual WFE is indeed very small, $10 \mathrm{~nm}$ for all possible Zenith angles.

Figure 5 shows the differential focus shift among the 6 LGS vs. Zenith angle, giving an indication of the required amount of differential re-focusing among different LGS probes.
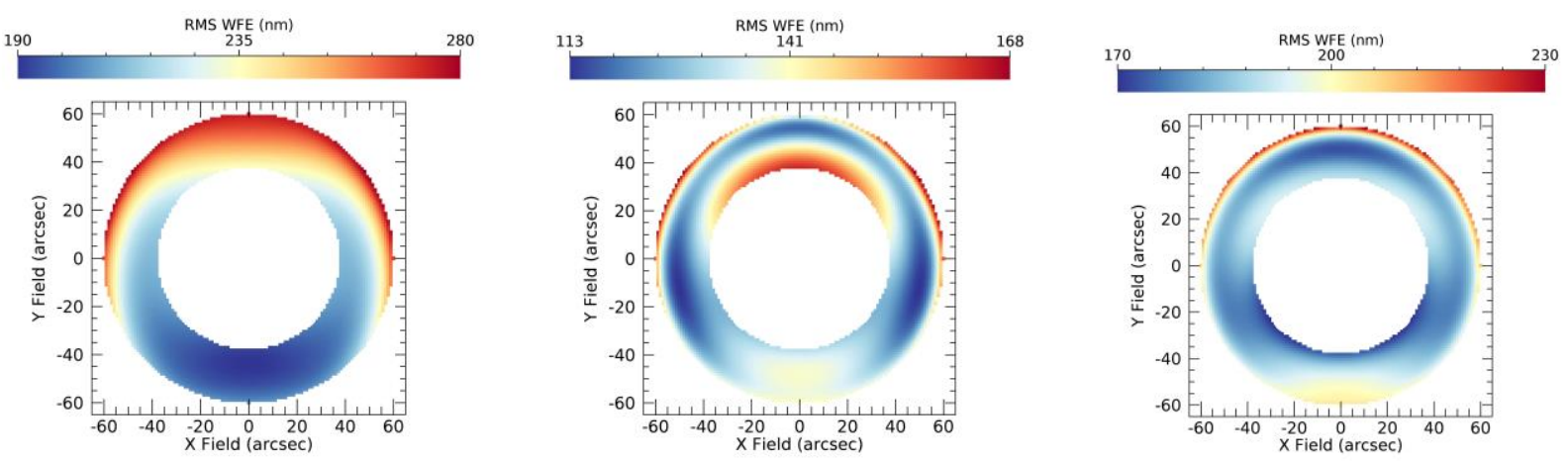

Figure 3. WFE maps at three different ranges of the LGS from the telescope. From left to right: $80 \mathrm{~km}-160 \mathrm{~km}-$ $240 \mathrm{Km}$. The WFE map is enclosed in a ring of inner diameter $45 \operatorname{arcsec}$ and outer diameter of 60 arcsec: these are the two possible cases of LGS asterism.

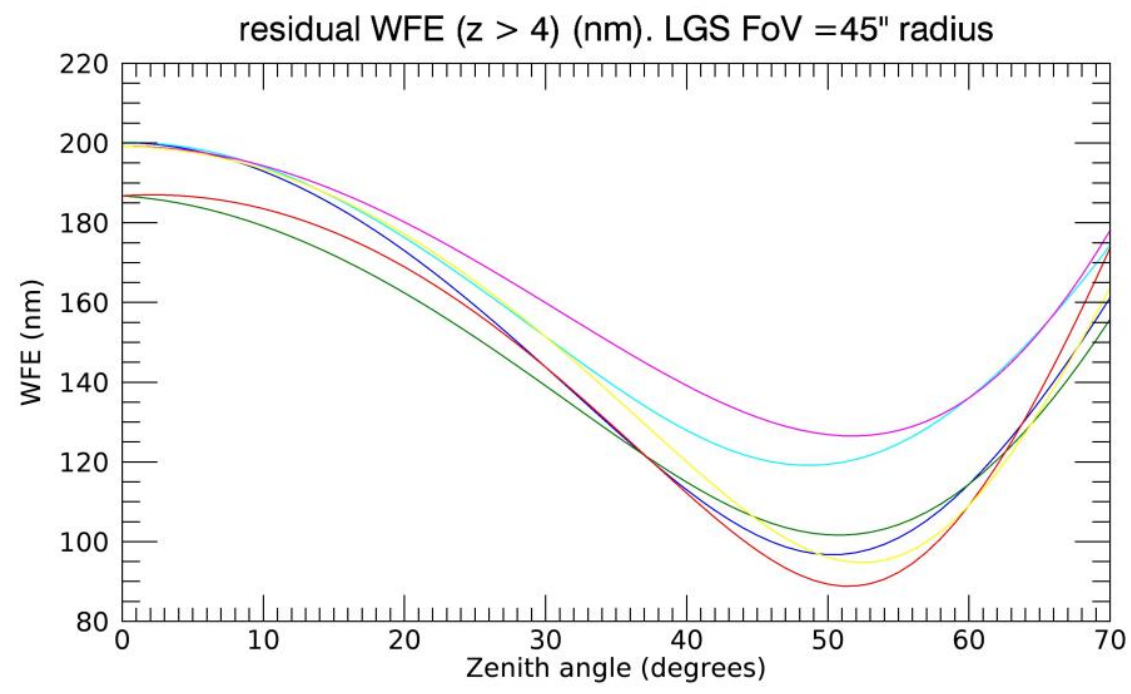

Figure 4. Residual WFE at the LGS Objective image plane (after removal of tip-tilt and focus) as a function of Zenith angle. The 6 curves correspond to an equal number of LGS, symmetrically arranged over a 45 arcsec radius FoV. 


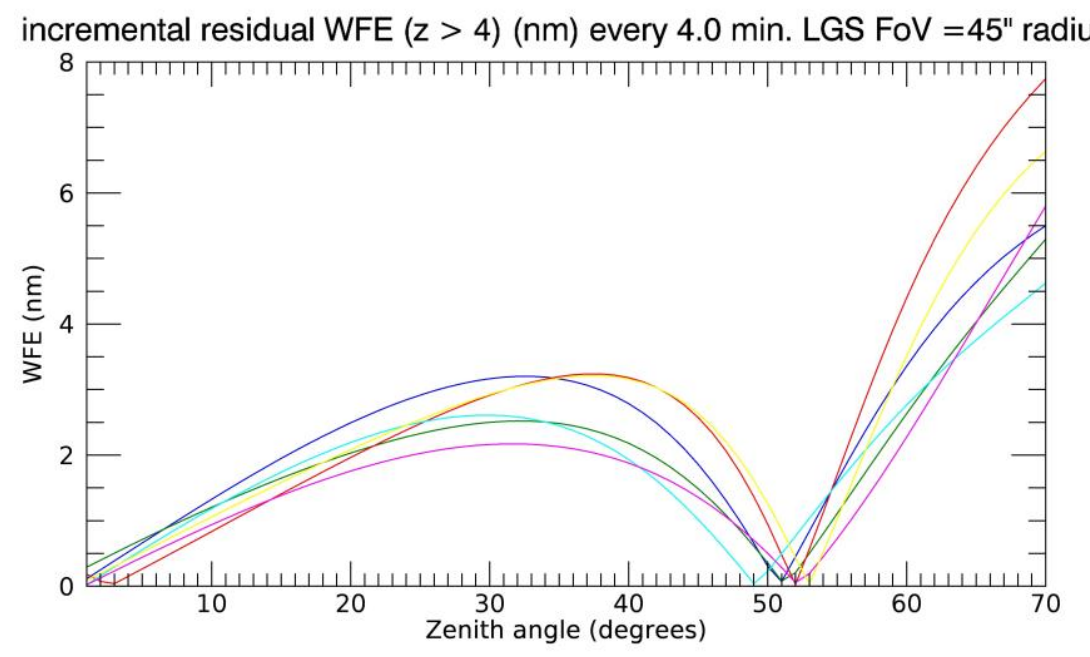

Figure 5. Variation of the residual WFE at the LGS Objective image plane (after removal of tip-tilt and focus) as a function of Zenith angle. The temporal average of the last 4 minutes is removed from the wavefront at each Zenith angle. The telescope tracks one degree every 4 minutes. The 6 curves correspond to an equal number of LGS, symmetrically arranged over a 45 arcsec radius FoV.

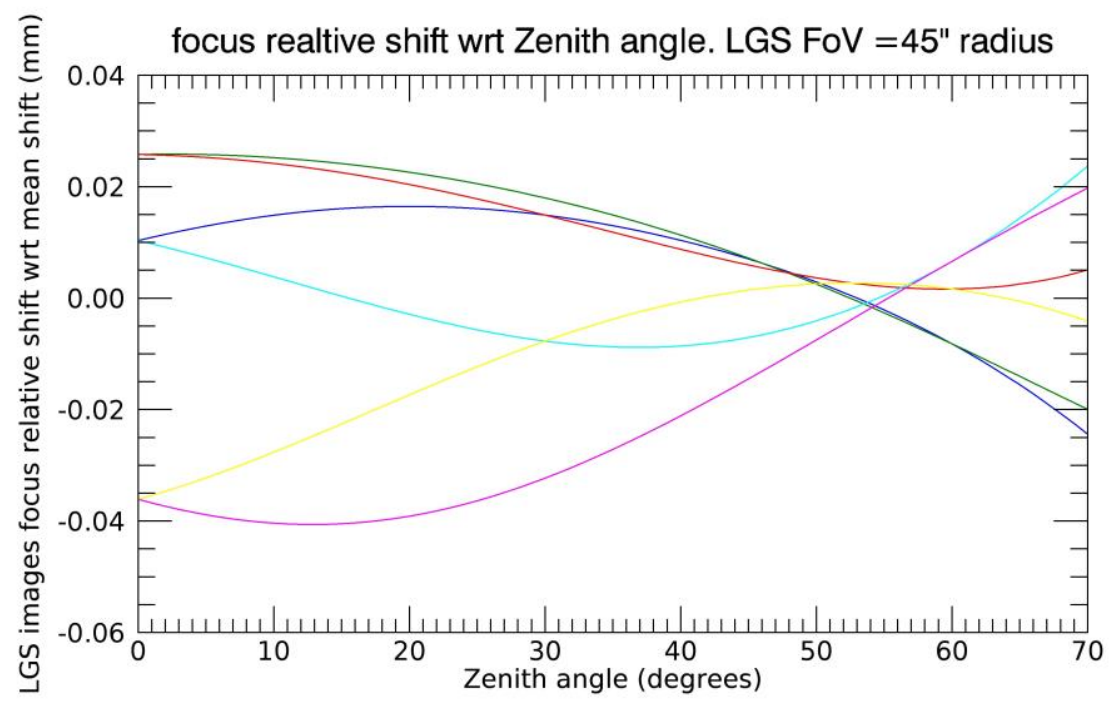

Figure 6. Differential focus position. LGS relative focus shift at the LGS Objective image plane as a function of Zenith angle. The 6 curves correspond to an equal number of LGS, symmetrically arranged over a 45 arcsec radius FoV.

\section{LGS OBJECTIVE TOLERANCE ANALYSIS}

The same approach which has been applied to the Main Path Optics [6] has also been used to tolerance the LGS Objective. The tolerance analysis has been performed with a limit of WFE degradation of about $30 \%$ and a pupil blur degradation of $1 / 10$ of sub-aperture (considering 80 sub-apertures along the diameter). The analysis has been split into three blocks:

- $\quad$ Block 1: Manufacturing errors;

- $\quad$ Block 2: Surface irregularities;

- Block 3: Alignment repeatability.

In the tolerance analysis, the main differences between the LGS Objective and the Main Path Optics are: 
- The LGS Objective contains lenses in the optical path;

- The RMS WFE of the LGS Objective shall exclude the TT and defocus terms;

- Geometric distortion is not an issue for the LGS Objective;

- LGSs are not focused at infinity but in a range of finite altitudes.

The next tables show the optical tolerances of the refractive elements in the LGS Objective.

\begin{tabular}{|l|r|}
\hline Parameter & Tolerance \\
\hline Curvature radius & $\pm 0.1 \%$ \\
\hline Conic constant & $\pm 0.05 \%$ \\
\hline Sag of flat surfaces (residual curvature) & $\pm 316 \mathrm{~nm}$ \\
\hline Wedge & $\pm 50 \mathrm{micro}-\mathrm{rad}$ \\
\hline Centering & $\pm 0.05 \mathrm{~mm}$ \\
\hline Surface irregularity RMS & $30 \mathrm{~nm}$ \\
\hline
\end{tabular}

Table 3. LGS Objective refractive elements general tolerances.

\begin{tabular}{|l|r|}
\hline Parameter & Tolerance \\
\hline Glass refractive index & $\pm 5 \mathrm{e}-4$ \\
\hline Glass Abbe number & $\pm 1 \%$ \\
\hline
\end{tabular}

Table 4. LGS Objective refractive elements material tolerances.

Mirror tolerances inside the LGS Objective are the same as for the mirrors in the Main Path Optics: the only exception is surface irregularity, which, in the case of the mirrors in the LGS Objective, is $30 \mathrm{~nm}$ RMS for all orders.

The sensitivities related to available DOF can be used during the alignment phase of the instrument since the worst offenders to system performance are also the best set of compensators for required adjustments. Motion ranges to compensate the tolerances of DOF and repeatability of chosen compensators are shown in Table 5.

Tolerance analysis results are shown through Monte Carlo simulations, where the figure of merit is the RMS WFE (without tip-tilt and defocus). 100 Monte Carlo trials were generated assuming Normal statistical distribution. The displayed values are for 6 LGSs at a radius of 45 arcsec, for 15 sampled Zenith angles.

Figure 7, Figure 8 and Figure 9 show the increment in WFE due to the computed tolerances for Block 1, Block 2 and Block 3 respectively. The results regarding the pupil blur degradation are compliant with the requirement, as showed in Figure 10.

\begin{tabular}{|c|c|}
\hline DOF used as compensators & Motion range to compensate tolerances of remaining DOF \\
\hline Axial position (Lenses $3-4-5$ - 6) & $\pm 30 \mathrm{~mm}$ \\
\hline X-Y Decenters (Lenses $2-3-5-6$ ) & $\pm 2 \mathrm{~mm}$ \\
\hline \multicolumn{2}{|c|}{ Tolerances of compensators (repeatability) } \\
\hline Axial position & $\pm 50 \mu \mathrm{m}$ \\
\hline X-Y Decenters & $\pm 30 \mu \mathrm{m}$ \\
\hline \multicolumn{2}{|c|}{ Tolerances of DOF } \\
\hline X-Y Decenters & $\pm 0.5 \mathrm{~mm}$ \\
\hline X-Y Tilts & $\pm 0.5 \mathrm{mrad}$ \\
\hline Axial Position & $\pm 0.5 \mathrm{~mm}$ \\
\hline
\end{tabular}

Table 5. LGS Objective refractive elements DOF tolerances. Some DOF (first 2 rows) are used as compensators to system misalignments. The tolerated misalignments are reported in the last 3 rows. The compensators must satisfy the repeatability requirements reported in the table. 

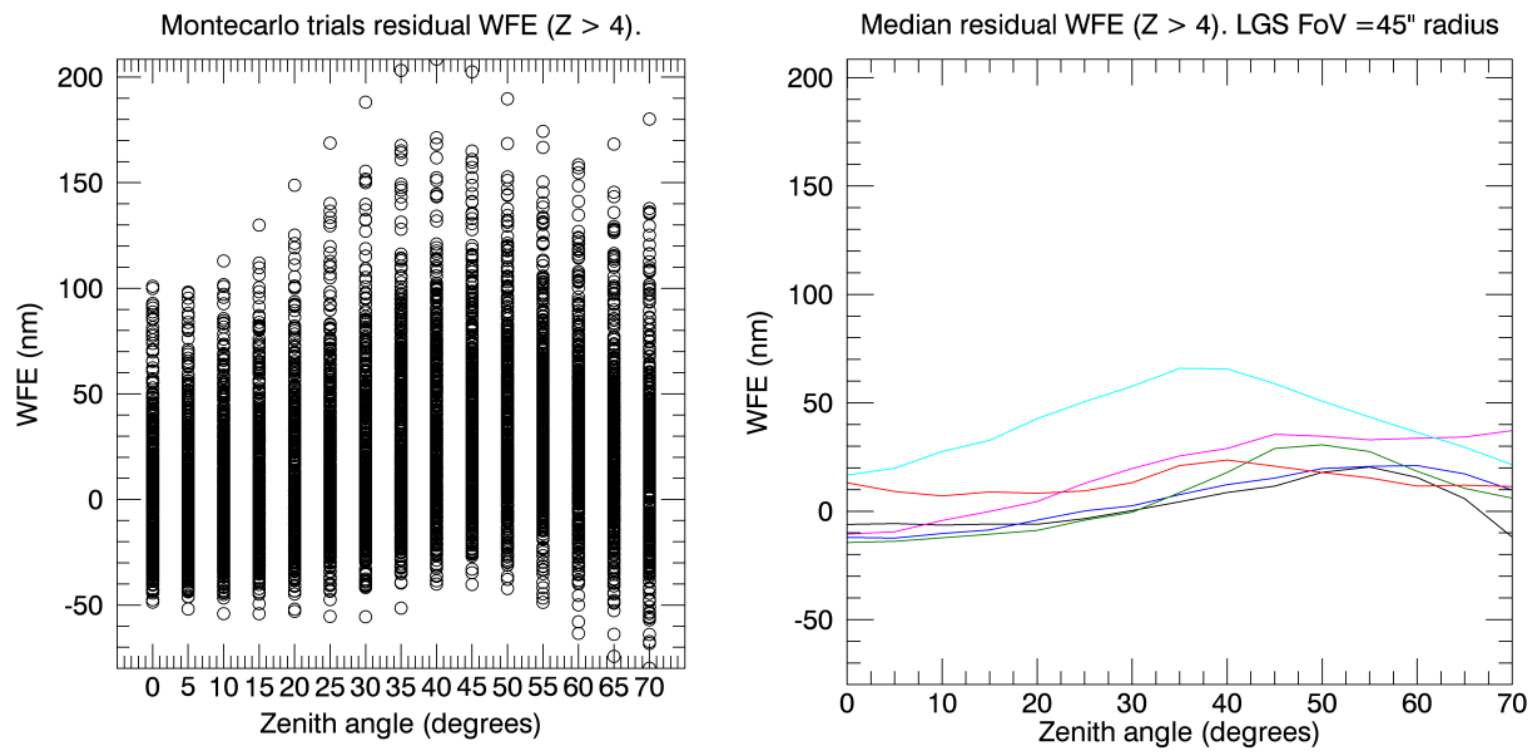

Figure 7. Monte Carlo trials for manufacturing errors of the LGS Objective optics. Left: variation of WFE at the LGS Objective image plane with respect to the nominal value as a function of Zenith angle. Right: median values of the left plot, different colours correspond to different positions around the 45 arcsec circle FoV of the LGSs.
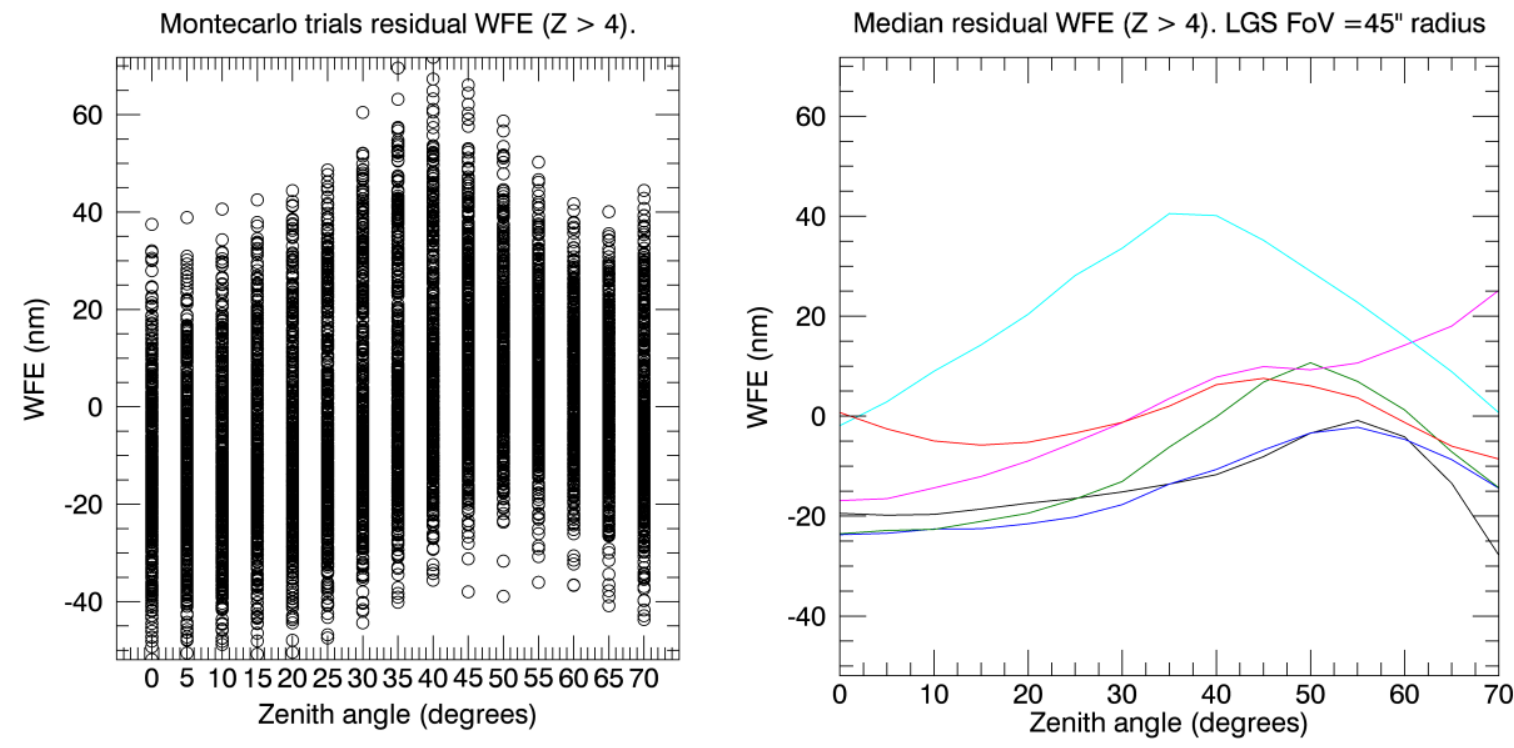

Figure 8. Monte Carlo trials surface irregularities of the LGS Objective optics. Left: variation of WFE at the LGS Objective image plane with respect to the nominal value as a function of Zenith angle. Right: Median values of the left plot, different colours correspond to different positions around the 45 arcsec circle FoV of the LGSs. 

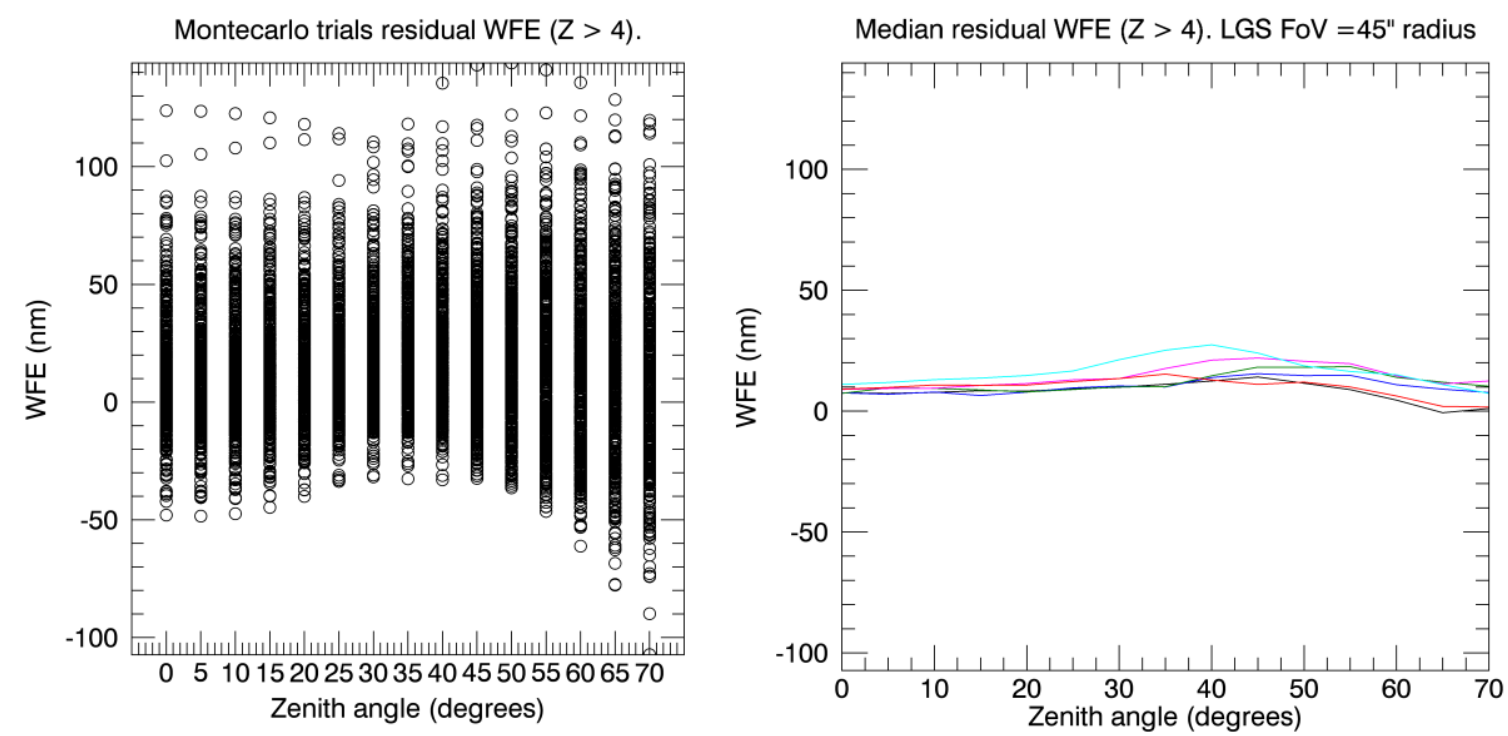

Figure 9. Monte Carlo trials for DOF tolerances. Left: variation of WFE at the LGS Objective image plane with respect to the nominal value as a function of Zenith angle. Right: Median values of the left plot, different colors correspond to different positions around the 45 arcsec circle FoV of the LGSs.

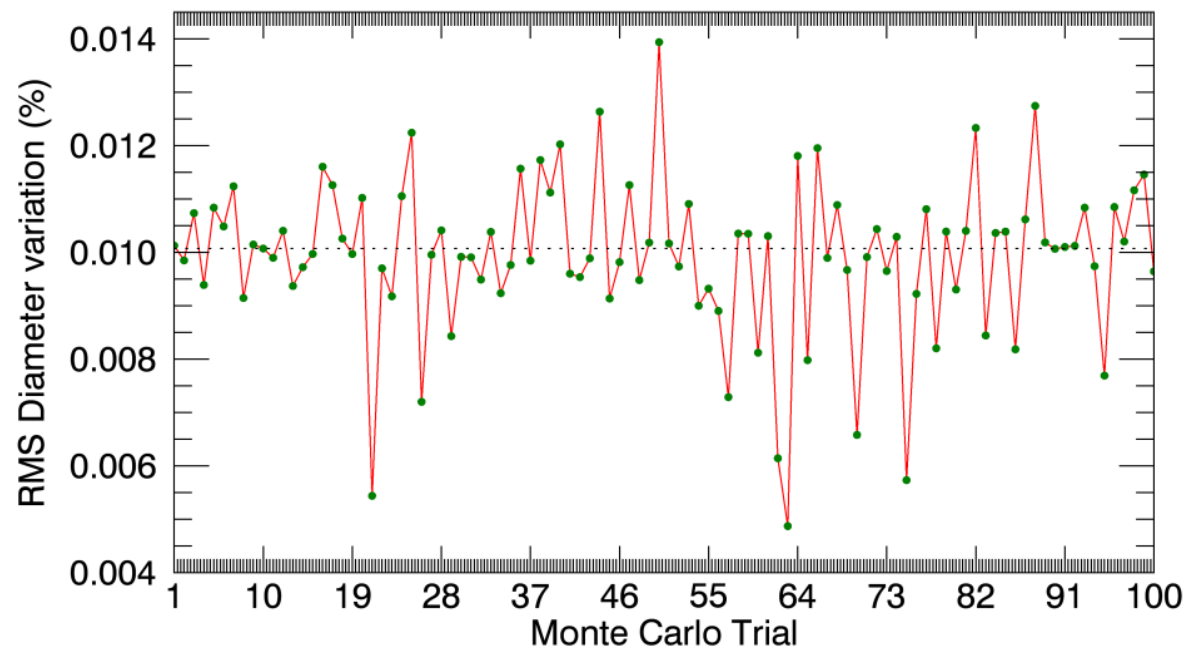

Figure 10. RMS of pupil image diameter variation (\%) inside the LGS Objective FoV due to the optical tolerances.

\section{CONCLUSIONS}

We presented the current design of the LGS Objective of MAORY. The residual quasi-static WFE is below 200nm for all Zenith angles. Moreover the variation with time of the WFE is very slow, allowing to average out the differential WFE respect the atmospheric one. At the time of writing some investigations are being carried on to see if a larger residual WFE is acceptable, in order to simplify the design and allow significant reduction of cost and possibly to easy the AIV procedure. The tolerance analysis shown that no particular issue should arise going from the nominal design to real components. 


\section{REFERENCES}

[1] Diolaiti, E., "MAORY: A Multi-conjugate Adaptive Optics RelaY for the E-ELT," The Messenger 140, 28-29 (2010).

[2] Diolaiti, E.; Arcidiacono, C.; Bregoli, G.; Butler, R. C.; Lombini, M.; Schreiber, L.; Baruffolo, A.; Basden, A.r; Bellazzini, M.; Cascone, E.; Ciliegi, P.; Cortecchia, F.; Cosentino, G.; De Caprio, V.; De Rosa, A.; Dipper, N.; Esposito, S.; Foppiani, I.; Giro, E.; Morgante, G.; Myers, R.; Patru, F.; Ragazzoni, R.; Riccardi, A.; Riva, M.; Zerbi, F. M.; Casali, M.; Delabre, B.; Hubin, N.; Kerber, F.; Le Louarn, M.; Marchetti, E.; Ramsay, S.; Stroebele, S.; Vernet, E.; "Preparing for the phase B of the E-ELT MCAO module project."; Proceedings of the SPIE, Volume 9148, id. 91480Y, (2014);

[3] Gilmozzi, R. and Spyromilio J., "The 42m European ELT: status," in Society of Photo-Optical Instrumentation Engineers (SPIE) Conference Series, Society of Photo-Optical Instrumentation Engineers (SPIE) Conference Series 7012, (2008).

[4] Davies, R.; Schubert, J.; Hartl, M.; Alves, J.; Clénet, Y.; Lang-Bardl, F.; Nicklas, H.; Pott, J.-U.; Ragazzoni, R.; Tolstoy, E.; Agocs, T.; Anwand-Heerwart, H.; Barboza, S.; Baudoz, P.; Bender, R.; Bizenberger, P.; Boccaletti, A.; Boland, W.; Bonifacio, P.; Briegel, F.; Buey, T.; Chapron, F.; Cohen, M.; Czoske, O.; Dreizler, S.; Falomo, R.; Feautrier, P.; Förster Schreiber, N.; Gendron, E.; Genzel, R.; Glück, M.; Gratadour, D.; Greimel, R.; Grupp, F.; Häuser, M.; Haug, M.; Hennawi, J.; Hess, H.-J.; Hörmann, V.; Hofferbert, R.; Hopp, U.; Hubert, Z.; Ives, D.; Kausch, W.; Kerber, F.; Kravcar, H.; Kuijken, K.; Lang-Bardl, F.; Leitzinger, M.; Leschinski, K.; Massari, D.; Mei, S.; Merlin, F.; Mohr, L.; Monna, A.; Müller, F.; Navarro, R.; Plattner, M.; Przybilla, N.; Ramlau, R.; Ramsay, S.; Ratzka, T.; Rhode, P.; Richter, J.; Rix, H.-W.; Rodeghiero, G.; Rohloff, R.-R.; Rousset, G.; Ruddenklau, R.; Schaffenroth, V.; Schlichter, J.; Sevin, A.; Stuik, R.; Sturm, E.; Thomas, J.; Tromp, N.; Turatto, M.; Verdoes-Kleijn, G.; Vidal, F.; Wagner, R.; Wegner, M.; Zeilinger, W.; Ziegler, B.; Zins, G. "MICADO: first light imager for the E-ELT", Proceedings of the SPIE, volume 9908 id 73, (2016);

[5] M. Lombini ; A. De Rosa ; P. Ciliegi ; F. Cortecchia ; E. Diolaiti ; Mauro Patti ; M. Bonaglia ; L. Busoni ; V. De Caprio ; S. Esposito ; P. Feautrier ; P. Rabou ; M. Riva ; E. Stadler; "Optical design of the post-focal relay of MAORY". Proc. SPIE 9908, Ground-based and Airborne Instrumentation for Astronomy VI, 9908AB (August 9, 2016);

[6] Patti M., Lombini M., Diolaiti E., Cortecchia F., Ciliegi F.;” Exploring MAORY performances through tolerance analysis"; This conference 